\title{
Conventional Dermatofibrosarcoma Protuberans
}

National Cancer Institute

\section{Source}

National Cancer Institute. Conventional Dermatofibrosarcoma Protuberans. NCI

Thesaurus. Code C49104.

A morphologic variant of dermatofibrosarcoma protuberans characterized by the presence of spindle-shaped fibroblastic cells, histiocytic cells, and a storiform growth pattern. 